\title{
The Impact of Social Media on Mobile Games: From the Participation and Communication of Players
}

\author{
Lingjun Xie \\ School of media and communication, The University of Sydney, Australia; \\ lxie9393@uni.sydney.edu.au
}

Keywords: social media; mobile games; connection; communication; participation

Abstract: Social media enable one person to communicate with thousands of other people [1]. The emergence of social media changes the cultural practice of games. Today, social media plays an increasingly important role in mobile games, and affects the communication between mobile gamers. This paper argues that social media has become a new method to disseminate mobile games, which can affect mobile gamers' communication, and improve players' participation and connection in the game. Firstly, this paper explains the definition of social media, and there is an overview introduction of how social media become a new method to disseminate mobile games, explores how social media influence game players' participation and communication from perspectives of social networking, game live streaming, and social advertising, and finally illustrates the concept of social ties and why social media can affect game players' participation and communication.

\section{Introduction}

Social media can be generally regarded as "Internet-based applications that carry user-generated content" [2]. By utilizing social media platforms, users can create content, share content, communicate with others, gain various information in the digital space. Social media plays an increasingly important role in social networking and content sharing [3]. Mobile games are supported by mobile devices and utilize mobile features such as portability (mobility), accessibility (generality), and convenience (simplicity) [4]. With the popularization of mobile devices, mobile games develop fast in recent years and more players are involved in mobile games. Nowadays, social media plays in increasing important role in disseminating mobile games. How does social media influence the spread of mobile games? This is a question that needs to be discussed from the characteristics of games.

An interesting phenomenon is that at the beginning of games, sociability was born with games. In the past, people tend to play traditional card games with their friends, and they are more likely to play these games with others than to play alone. With the advent of the internet, people start to play computer games, but they are not satisfied with only interact with machines. The development of social media coupled with mobile games has roused players' interests. In addition, there are many connections between mobile games and social media. Both of them are the products of the internet era. Social media also influence the cultural practice of mobile games. For example, the 
combination of social media and mobile games form a new genre of mobile games, which is called social network game. Social network game is a social game based on the online social network [5]. It is mentioned that these games involved with social connections "have multiplied like wildfire on social networking sites" [6]. Users can play these games with their friends on social networking, or they can also invite their friends to the game. The concept of social network game is evolved with the development of social network and mobile games, but these games have some common characteristics. A Social Network Game "is a video game satisfying the criteria of asynchronous play, casual multiplayer, coopetition, and beneficial social media interaction” [7]. For example, Farmville is one the most popular social network games with more than 80 million users on the social networking site Facebook [8].

Social media has many forms, live streaming is also a genre of social media where users can create content and communicate with others. Video streaming was previously at the fringes of social media, but game live streaming began growing in popularity since 2009 [9]. Players can watch game video, game live and communicate with others in the game live platform. In the past, players who have same interests can be gathered in a forum, but game live streaming is an active medium that provides gamers with a new form of communication. For example, Twitch is a live video streaming platform where gamers can broadcast their gameplay [10]. In addition, Twitch provides a platform for gamers to communicate, which foster a participatory community for mobile gamers.

Social media play an increasingly important role in the dissemination of mobile games, which affect players' participation and communication. Examples of Facebook and Farmville, Tencent and King of glory, Twitch can help to illustrate how social media improve mobile gamers' participation and increase their communication.

\section{Social Media Provide Social Interaction for Players}

Farmville is a "real-time market-based in which players have the task to manage a virtual farm by planting" [11]. In fact, the game model is simple and friendly for new beginners. It runs based on Facebook and Facebook users can play the game with real friends. The game model is simple, Facebook users have a virtual farm that can grow and harvest crops, trees and animals and users can visit other users' farms [12].

When mobile games combine with social media, more users are motivated to participate in the game and their communication is strengthened in the process. Entertainment, competition, challenge, social interaction and social contact are general motivations that can encourage people to play mobile games [13]. According to this, it is plausible to conjecture that people tend to play games because they are motivated by these factors. As a social networking platform, Facebook provides a digital space for users to share information, create content, communicate with friends. Facebook is a main social platform that facilitates social interaction and conversation, and it is becoming a venue for game players because of its collaborative and social characteristics [14]. Facebook can provide social interaction for players in Farmville and social interaction is one of the most important motivations that encourage people to participate in the mobile game.

Facebook provides an explicit method to recruit more players: friends receive updates of the user's activity in the game and they will be introduced to the game[15]. Players can invite their friends to the game, and they can compete with their friends in the game, and they can be motivated

to engage in the Farmville because they can compete with their friends. For example, they can decorate their farms and compare with others' farm. By competing with others, players will gain a sense of fulfillment.

Self-presentation is an important reason why people like playing social networking games [16]. In some traditional video games, players can't present themselves to their social friends, but 
Farmville is a game providing a platform where players can present themselves to their social friends. Farmville allows players to create their new identifies in a virtual world and manage their impression in the platform, in addition, these new identities and impression can be presented to their friends in real life. Players can help their friends fertilizer, which will leave a good impression on their friends. In addition, lots of activities in the game can strengthen the communication between players, like visiting friends' farm, sending gifts to their friends. These interactive activities can increase communication, strengthen their personal communication both in the virtual and real world. In the meanwhile, these interactive activities can cultivate social contact and meet players' requirements of contacting with their social friends.

Social networking can also influence other genres of mobile games, like MOBA game. Multiplayer online battle arena (MOBA) games have become one of the most popular game genres, which has attracted millions of players in recent years [17]. Why are so many players engaged in MOBA? The social factor is one of the most important reasons for its popularity [18]. Social media can motivate players to participate in the game and improve their personal communication. Take Tencent's King of glory as an example, players of the King of glory can log in the game by using WeChat, then invite their friends to their team and compete with others. Players' social friends in social networking will be introduced into the mobile game. The relationship between WeChat and King of glory seems like Facebook and Farmville. More game players will be motivated to participate in the game and play it with their social friends. What's more, social networking is a platform that can advertise and market the game, which is becoming a new method of spreading mobile games.

To sum up, people will participate in a game because of motivations, including social interaction, social contact, competition, self-presentation. Mobile games, combined with social networking can evoke players' motivation to participate in the game. Social networking provides a platform for mobile game players to interact and socialize with their friends, compete and corporate with social friends, and present themselves in the front of others. Players' participation in the game can be improved because of these motivations. In addition, through interactive activities in the game, the communication between players is becoming more frequent and people are not playing alone in the mobile game.

\section{Social Media Create Virtual Communities for Players}

Live streaming has emerging as a growing genre of participatory social media [19]. In recent years, game live streaming is gaining popularity among game players and the platforms of game live have proliferated. The case study of Twitch can explore how game live streaming affect players' participation and communication in mobile games. As one of the most popular game live platforms, Twitch allows users to share game videos, create content, and broadcast their game videos to public audiences [20]. Twitch is a bridge between viewers and gamers, providing a platform for them to interact with each other.

One of the obvious characteristics of Twitch is building a community for players. Players may have initial interests in the mobile game, and such a game community can be regarded as external stimuli that encourage players to be more engaged in the game. In addition, viewers who have similar interests will be gathered in the platform, and communicate with each other. Many game streams, like Twitch, are fostering participatory communities and these open communities will encourage viewers to participate in mobile games[21]. According to this, players who enter Twitch will form their community, and community culture is important for game players. Before entering such a community, players may not have a sense of affiliation, but they can find their organization, or community through game live streaming. Additionally, they can present themselves in the 
community. With these factors, the participatory community can improve players participation in mobile games.

Another reason why Twitch can improve players' participation and communication in mobile games is about sociability. According to the research on Twitch users, it is found that tension release, social integrative are two distinct motivations why people like watching game videos in Twitch[22]. When viewers are watching videos on the platform, they can be attracted in various games, and they can also interact with streamers or other players. Their initial interests in mobile games can be cultivated by watching game videos, and they can also form their own game communities. Viewers may be unfamiliar with each other at the beginning, but they will get to know others via the social community created by Twitch. Their connection and communication will be enhanced by such a platform, which will encourage players to participate in the mobile game.

"games are inherently active medium, without interaction a game is benign"[23]. According to this, interaction is quite important for mobile games. Game players can comment, share content or broadcast their game lives and present themselves in the platform. They can interact with others in game live streaming. Interaction can be one of the most important reasons why the game live streaming can be a new method of propagating mobile games and influence players' participation and communication.

Social media, like gave live streaming can create a virtual community for players. People who like playing mobile games will be gathered in the community. They will find a space they can communicate, share, chat and play together. Their interaction and communication will be developed when they enter such a community and they will be encouraged to participate in the game. Without these game communities, it is difficult for people to find a method to interact with other players, and they may lose interests in the game, therefore their participation in the game will be reduced. Players' relationship and participation in the game can be developed in the game community.

\section{Game Advertising on Social Media}

Mobile game is a virtual world where players have power to make friends, real-time interact with others, present themselves, accomplish mission with others, and the most important function of mobile games is "Internet communication functions"[24]. Players would like to play games with their friends, so that's why social network games can be so popular in the world. However, without the supporting of social media, players are unable to present themselves and they cannot interact with their social friends, thus the game community cannot be formed. Social media, like social networking and game live streaming, can improve players' enthusiasm in game play. In fact, social media redefine the traditional way of game play, and create a new interaction method for players. Therefore, their participation in the game will be improved. Social media also can be a platform where players can communicate with others. The analysis of social networking and game live streaming can illustrate how social media influence game players' participation and communication in mobile games.

Advertising in social media can be another way that diffuses mobile games. Social media provide unique platforms for game advertisers. Marketers and advertisers will locate their brands in social media [25]. Interactive advertising on social media can help to promote the game and attract more players. For example, game advertisements on Facebook or Twitter will be recommended to related users, which can be called precision marketing. Another example is that attractive and vivid advertisement can be shown in YouTube videos to draw users' attention. Digital advertising methods emerge with the development of social media, which will appeal to players and drive them to engage in the mobile game. Advertising in social media can also improve players' communication. When users post their game screenshots of new levels they achieved on social 
media, users with similar interests can communicate. The consumer-to-consumer influence in social media may impact users' attitudes and behaviors [26]. Game advertising can be another method that affects game play, and more players can be influenced by game advertising in social media.

\section{Weak Ties and Strong Ties}

Relationship in social media can be characterized as two types: strong ties and weak ties, strong ties are those who "you really trust, people whose social circles tightly overlap with your own", while weak ties refer to "merely acquaintances" [27]. Strong ties and weak ties are two opposite concepts that can be presented in various social media platforms. The relationship or connection between players in mobile games maybe not as strong as their relationship in social networking. In mobile gameplay, all of activities can be done anonymously.

It is undeniable that that the relationship in real world and virtual world is different. When people start to play mobile games with their friends in social networking, the strong ties in social networking can be transferred into mobile games. Their social interaction can be improved when playing mobile games and more players will be motivated to participate the games. In the same way, with game live streaming, players can create virtual communities, their social ties can be strengthened since their social interaction in the virtual organization. Community can increase social conformity, which will influence players' behavior and impact how a consumer engages in online communities [28]. According to this, the game community can increase social conformity of players, and impact their participation in the game. In addition, when the ties and social conformity between players are becoming strong, their communication will also be promoted.

\section{Conclusion}

Social media has changed the cultural practice of mobile games, including the way of transmitting mobile games, the relationship between game players, and the method of playing games. From players' participation and communication perspective, social media can encourage players to participate in the mobile games and it creates strong social ties for game players. Social media can meet game players' requirements of presenting themselves in the front of social friends and interacting with them. Social media can be regarded as a unique platform that has profound influence on players' engagement. In addition, visual communities can be created in the process to improve players' communication. Social media also create new game advertising methods to attract more players and inspire them to engage in the game. Because of these characteristics, social media will have a profound influence in mobile games and game players.

\section{References}

[1] Mangold W G, Faulds D J. Social media: The new hybrid element of the promotion mix[J]. Business horizons, 2009, 52(4): 357-365.

[2] Blackshaw P. The consumer-generated surveillance culture[J]. Retrieved August, 2006, 17: 2011.

[3] Asur S, Huberman B A. Predicting the future with social media[C]//Proceedings of the 2010 IEEE/WIC/ACM International Conference on Web Intelligence and Intelligent Agent Technology-Volume 01. IEEE Computer Society, 2010: 492-499. IEEE/WIC/ACM International Conference on (Vol. 1, pp. 492-499). IEEE.

[4] Jeong E J, Kim D J. Definitions, key characteristics, and generations of mobile games[M]//Mobile computing: Concepts, methodologies, tools, and applications. IGI Global, 2009: 289-295.

[5] Konert J, Söbke H, Wendel V. Social Network Games[M]//Entertainment Computing and Serious Games. Springer, Cham, 2016: 442-474.

[6] Shin D H, Shin Y J. Why do people play social network games? [J]. Computers in Human Behavior, 2011, 27(2): 852-861.

[7] Konert J. Interactive Multimedia Learning: Using Social Media for Peer Education in Single-Player Educational 
Games[M]. Springer, 2014.

[8] Cashmore P. Farmville surpasses 80 million users[J]. Retrieved October, 2010, 15: 2013.

[9] Hamilton W A, Garretson O, Kerne A. Streaming on twitch: fostering participatory communities of play within live mixed media[C]//Proceedings of the SIGCHI Conference on Human Factors in Computing Systems. ACM, 2014: $1315-1324$.

[10] Pires K, Simon G. Dash in twitch: Adaptive bitrate streaming in live game streaming platforms[C]//Proceedings of the 2014 Workshop on Design, Quality and Deployment of Adaptive Video Streaming. ACM, 2014: 13-18.

[11] Griffiths M D. Gaming in social networking sites: a growing concern? [J]. World Online Gambling Law Report, 2010, 9(5): 12-13.

[12] Reporter S. Facebook farmers want India flag. BBC News[J]. 2009.

[13] Schuurman D, De Moor K, De Marez L, et al. Fanboys, competers, escapists and time-killers: a typology based on gamers' motivations for playing video games[C]//Proceedings of the 3rd international conference on Digital Interactive Media in Entertainment and Arts. ACM, 2008: 46-50.

[14] Burroughs B. Facebook and FarmVille: A digital ritual analysis of social gaming[J]. Games and Culture, 2014, 9(3): 151-166.

[15] Wei X, Yang J, Adamic L A, et al. Diffusion Dynamics of Games on Online Social Networks[C]//WOSN. 2010.

[16] Mehdizadeh S. Self-presentation 2.0: Narcissism and self-esteem on Facebook[J]. Cyberpsychology, behavior, and social networking, 2010, 13(4): 357-364.

[17] Nosrati M, Karimi R, Hariri M. General trends in multiplayer online games[J]. World Applied Programming, 2013, 3(1): 1-4.

[18] Ducheneaut $N$, Yee $N$, Nickell E, et al. Alone together? exploring the social dynamics of massively multiplayer online games[C]//Proceedings of the SIGCHI conference on Human Factors in computing systems. ACM, 2006: 407-416.

[19] [23]. Smith T, Obrist M, Wright P. Live-streaming changes the (video) game[C]//Proceedings of the 11th european conference on Interactive TV and video. ACM, 2013: 131-138.

[20] Pan R, Bartram L, Neustaedter C. TwitchViz: a visualization tool for twitch chatrooms[C]//Proceedings of the 2016 CHI Conference Extended Abstracts on Human Factors in Computing Systems. ACM, 2016: 1959-1965.

[21] Sjöblom M, Hamari J. Why do people watch others play video games? An empirical study on the motivations of Twitch users [J]. Computers in Human Behavior, 2017, 75: 985-996.

[22] Lo S K, Wang C C, Fang W. Physical interpersonal relationships and social anxiety among online game players[J]. Cyberpsychology \& behavior, 2005, 8(1): 15-20.

[23] Okazaki S, Taylor C R. Social media and international advertising: theoretical challenges and future directions[J]. International marketing review, 2013, 30(1): 56-71.

[24] Chu S C, Kim Y. Determinants of consumer engagement in electronic word-of-mouth (eWOM) in social networking sites [J]. International journal of Advertising, 2011, 30(1): 47-75.

[25] Gilbert E, Karahalios K. Predicting tie strength with social media[C]//Proceedings of the SIGCHI conference on human factors in computing systems. ACM, 2009: 211-220.

[26] Venkatesh V, Morris M G. Why don't men ever stop to ask for directions? Gender, social influence, and their role in technology acceptance and usage behavior [J]. MIS quarterly, 2000: 115-139. 